\title{
Systèmes de santé européens: I'herbe est-elle vraiment plus verte chez nos voisins?
}

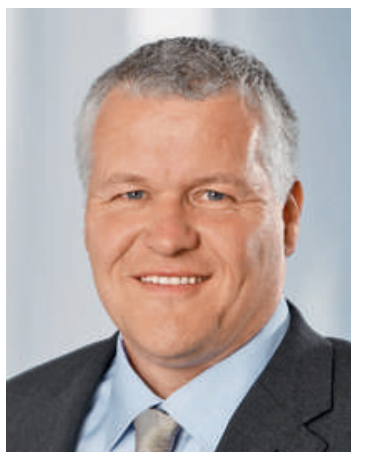

Portons notre regard vers l'ouest et comparons notre système de santé à celui de la France. En ce qui concerne la répartition des tâches entre médecins de premier recours et spécialistes, les deux pays font jeu égal: en principe, ils distinguent les prestations hospitalières des prestations ambulatoires, ces dernières étant majoritairement fournies dans le secteur ambulatoire non public. L'Allemagne, la Belgique et l'Autriche connaissent des structures similaires. En Suisse, le taux de médecins exerçant dans le secteur ambulatoire est de 53,1\%. En France il s'élève à 46,6\%. La proportion des coûts totaux de la santé dans le produit national brut est également comparable (11\% en CH et $11,6 \%$ en F).

Cependant, des divergences significatives apparaissent en matière d'accès aux soins: en Suisse, $92 \%$ des patients sont examinés dans le mois par un spécialiste, en France, ils ne sont que $67 \%$. Cette différence persiste en médecine de premier recours: une consultation souhaitée en urgence a lieu dans $81 \%$ des cas le même jour en Suisse alors qu'en France ce n'est possible que dans 59\% des cas. En soins aigus, les écarts

\section{Un système étatique n'est pas synonyme d'un meilleur flux d'informations entre hôpitaux et médecins traitants.}

sont moins perceptibles, comme le montrent les taux de létalité après un infarctus du myocarde $(5,9 \%$ en $\mathrm{CH}$ contre $6,2 \%$ en $\mathrm{F}$ ) ou un AVC (7,0\% en $\mathrm{CH}$ contre $8,5 \%$ en $\mathrm{F}$ ). Mais le nombre de lits d'hôpitaux de soins aigus pour 100000 habitants est 15,7\% inférieur en Suisse (320, contre 380 en F). Notons toutefois que les deux pays affichent une tendance similaire à vouloir les réduire.

On pourrait croire que le flux d'informations entre les hôpitaux et les médecins traitants devrait être nettement plus performant (réseau) dans un système de santé étatique organisé de manière relativement centralisée (Agence régionale de la santé, ARS). Mais ce n'est pas le cas. Lors d'un suivi posthospitalier, seuls 10\% de nos confrères en France reçoivent une information détaillée dans les 48 heures suivant la sortie du patient. La Suisse ne se place pas à la meilleure place mais ce taux s'élève quand même à $40 \%$ chez nous. En matière de productivité, un médecin de famille en Suisse consacre environ 15,6 minutes à une consultation contre 22,2 minutes en France, soit $42,3 \%$ de temps en plus. En revanche, il est frappant de constater que 91,3\% des sondés en Suisse estiment que la durée de consultation est suffisante alors qu'en France, ils ne sont «que» $85,4 \%$.

Il est également intéressant de voir que la rémunération des prestations, mesurée en parité de pouvoir d'achat, se comporte à l'inverse de l'impression générale: chez les médecins de premier recours français, elle est inférieure de 23,3\% à celle de leurs confrères en Suisse. Chez les spécialistes, c'est le contraire, les confrères en Suisse se situent 19,4\% plus bas qu'en France [1].

\section{Pour les patients, le système de santé helvétique est plus efficace que le système français.}

En Suisse, 69\% de la population indique être satisfaite de son système de santé alors que seuls $40 \%$ de nos voisins partagent cette opinion. Autre fait surprenant: les patients en France paient de leurs poches les factures inférieures à 100 \$ dans $42,1 \%$ des cas [2], bien que tous les citoyens soient affiliés à la sécurité sociale et que près de $95 \%$ d'entre eux aient contracté une assurance supplémentaire («mutuelle» d'intérêt public ou complémentaire privée). En Suisse, ce taux pour les factures n'est «que» de 26,8\%. En outre, les assurances en France refusent de prendre en charge les coûts de santé dans $17 \%$ des cas, taux légèrement supérieur à celui de la Suisse qui se situe à $15,7 \%$.

Un système à forte régulation étatique ne garantit donc ni l'équité sociale ni une satisfaction des patients / assurés supérieure à la moyenne. Au contraire, un domaine de la santé très réglementé induit un recul de la productivité. En matière de santé, l'herbe n'est donc pas plus verte en France.

Dr Remo Osterwalder, membre du Comité central de la FMH, responsable du département Médecins en libre-pratique

1 Base de données de l'OCDE sur la santé 2008.

2 Commonwealth Fund, International Health Policy Survey 2013. 